\title{
Efetividade do ácido tranexâmico em pacientes sistemicamente comprometidos na odontologia: Revisão integrativa
}

Effectiveness of tranexamic acid in patients systemically compromised in dentistry: An integrative review

Efectividad del ácido tranexámico en pacientes sistémicamente comprometidos en odontología: Una revisión integradora

Emerson de Sousa Pinheiro ORCID: https://orcid.org/0000-0003-3844-470X Universidade de Brasília, Brasil E-mail: emersonpisousa@gmail.com

Kátia Maria Martins Veloso ORCID: https://orcid.org/0000-0002-0496-286X Universidade Federal do Maranhão, Brasil E-mail: kmmv69@hotmail.com

Sâmia Moreira de Andrade ORCID: https://orcid.org/0000-0002-2310-2515 Centro Universitário Santo Agostinho, Brasil E-mail: samia.andrade27@hotmail.com Evaldo Hipólito de Oliveira ORCID: https://orcid.org/0000-0003-4180-012X Universidade Federal do Piauí, Brasil E-mail: evaldohipolito@gmail.com

\begin{abstract}
Resumo
O ácido tranexâmico é uma substância inibitória da ação da enzima plasminogênio, que normalmente se liga aos coágulos para destruí-los. No entanto, em pessoas com doenças sistêmicas, o plasminogênio também pode impedir a formação de coágulos, dificultando a cessação de hemorragias. Suas formulações terapêuticas incluem as formas de comprimido, gel ou sob forma de injetável para corrigir ou evitar emergências relacionadas com sangramentos excessivos, sendo assim, um agente eficaz para hemostasias locais em procedimentos odontológicos invasivos. Este estudo tem o objetivo de demonstrar a atual utilização do ácido tranexâmico nos diversos procedimentos odontológicos evidenciando sua eficácia no processo clínico-cirúrgico. Trata-se de uma revisão bibliográfica integrativa realizada através de levantamento bibliográfico nas principais bases eletrônicas de dados, SciELO, LILACS, MEDLINE e BBO consultando-se a literatura disponível no período de 2009 a 2019. Observou-se que o tratamento imediato com ácido tranexâmico reduz o risco de morte em $70 \%$ em pessoas com sangramento grave. No entanto, o início do tratamento rápido é importante, pois mesmo pequenos atrasos estão associados a reduções no benefício. O ácido tranexâmico é indicado, principalmente, para reduzir o risco de hemorragia durante cirurgias e tratar hemorragias associadas à fibrinólise excessiva, mostrando-se seguro e efetivo nos procedimentos odontológicos de pacientes sistemicamente comprometidos, tais como as cirurgias orais menores, reparos ósseos periimplantares, implantes dentários, manejo de lesões císticas e raspagem subgengivais, dentre outros.
\end{abstract}

Palavras-chave: Ácido tranexâmico; Doenças sistêmicas; Odontologia.

\section{Abstract}

The tranexamic acid is a substance that inhibits the action of plasminogen, which normally binds to clots to destroy them. However, in people with systemic diseases, plasminogen can also prevent clots from forming, making it difficult to stop bleeding. Its therapeutic formulations include tablet, gel or injectable forms to correct or prevent emergencies related to excessive bleeding, thus being an effective agent for local hemostasis in invasive dental procedures. This study aims to demonstrate the current use of tranexamic acid in various dental procedures, highlighting its effectiveness in the clinical and surgical process. This is an integrative bibliographic review conducted through a bibliographic survey in the main electronic databases, SciELO, LILACS, MEDLINE and BBO consulting the available literature from 2009 to 2019. Immediate treatment with tranexamic acid was found to reduce the risk of death by $70 \%$ in people with severe bleeding. However, early treatment is important because even small delays are associated with reductions in benefit. Tranexamic acid is indicated primarily to reduce the risk of bleeding during surgery and to treat bleeding associated with excessive fibrinolysis, and is safe and effective in the dental procedures of systemically compromised 
patients, such as minor oral surgeries, periimplant bone repairs, implants. dentistry, management of cystic lesions and subgingival scraping, among others.

Keywords: Tranexamic acid; Systemic diseases; Dentistry.

\section{Resumen}

El ácido tranexámico es una sustancia que inhibe la acción de la enzima plasminógeno, que normalmente se une a los coágulos para destruirlos. Sin embargo, en personas con enfermedades sistémicas, el plasminógeno también puede prevenir la formación de coágulos, lo que dificulta detener el sangrado. Sus formulaciones terapéuticas incluyen tabletas, gel o formas inyectables para corregir o prevenir emergencias relacionadas con el sangrado excesivo, siendo así un agente eficaz para la hemostasia local en procedimientos dentales invasivos. Este estudio tiene como objetivo demostrar el uso actual del ácido tranexámico en diferentes procedimientos dentales, mostrando su efectividad en el proceso clínico-quirúrgico. Se trata de una revisión bibliográfica integradora realizada a través de un relevamiento bibliográfico en las principales bases de datos electrónicas, SciELO, LILACS, MEDLINE y BBO, consultando la literatura disponible de 2009 a 2019. Se observó que el tratamiento inmediato con ácido tranexámico reduce el riesgo de muerte en un $70 \%$ en personas con hemorragia grave. Sin embargo, el inicio de un tratamiento rápido es importante, ya que incluso los pequeños retrasos se asocian con reducciones en el beneficio. El ácido tranexámico está indicado, principalmente, para reducir el riesgo de hemorragias durante las cirugías y para tratar las hemorragias asociadas a la fibrinólisis excesiva, demostrando ser seguro y eficaz en los procedimientos dentales de pacientes sistémicamente comprometidos, como cirugías bucales menores, reparaciones óseas periimplantarias, implantes dentales, manejo de lesiones quísticas y raspado subgingival, entre otros.

Palabras clave: Ácido tranexámico; Enfermedades sistémicas; Odontología.

\section{Introdução}

O ácido tranexâmico é um medicamento utilizado para neutralizar o sistema de fibrinólise durante o processo de cicatrização em qualquer região do organismo. Esse processo de destruição do coágulo de fibrina acontece com mais facilidade, principalmente, em pacientes que possuem discrasias sanguíneas, uma vez que a cascata de coagulação não ocorre de maneira suficientemente adequada gerando assim alterações hematológicas como, por exemplo, as hemorragias (Ferrari et al., 2015; Nematullah et al., 2009).

O mecanismo de ação do ácido tranexâmico ocorre na fase posterior à formação do coágulo, agindo assim no aumento do tempo de dissolução da rede de fribina, por isso é classificado farmacologicamente como um antifibrinolítico (O'Leary et al., 2009).

Nesse sentido, o ácido não é um agente de ativação da cascata de coagulação, haja vista que sua ação é preservar o coágulo, gerando um processo hemostático eficiente, consequentemente, reduzindo a intensidade e os riscos de sangramento (Ferrari et al., 2015; O'Leary et al., 2009).

Dessa forma, sabe-se que corriqueiramente pacientes com necessidades especiais e/ou sistemicamente comprometidos buscam cada vez mais o atendimento odontológico devido à carência de cuidados bucais, gerando assim a indispensabilidade de procedimentos muitas vezes invasivos. Porém, os mesmos apresentam com frequência doenças que desencadeiam alterações sistêmicas que estão estreitamente relacionadas com problemas na cicatrização, dentre elas estão: leucemia, hemofilia, anemia falciforme, diabetes, doença de Von Willebrand, síndrome de Bernard Soulier, Tromblastenia de Glazmann, etc (Farrkh et al., 2016; Kuhn-Dall’Magro, 2011).

Assim sendo, a indicação do ácido tranexâmico na odontologia é usualmente prescrita de várias formas farmacológicas, desde comprimido para agir de forma sistêmica assim como também em gel tópico após cirurgias orais para prevenir que o coágulo seja desconstruído ${ }^{1}$. Nesse contexto, este agente antifibrinólitico tem se mostrado como uma grande droga de efeito hemostático bucal, pois se apresenta bastante consistente na manutenção do local de ação, evidenciando a sua efetividade como um material pró-coagulante (Ferrari et al., 2015; Ripollés-de Ramón et al., 2014).

Atualmente, o ácido tranexâmico é um dos três medicamentos mais empregado para pacientes com dificuldades de coagulação tanto na área cirúrgica médica como odontológica, isso por que além de ser seis a dez vezes mais potente do que o ácido aminocapróico, possui aspectos primordiais para sua eficácia, que são: afinidade pelo plasminogênio bem como maior 
atividade antifibrinolítica devido seu bom tempo de ação (Nematullah et al., 2009; O'Leary et al., 2009).

Diante disso, este estudo foi criado devido a necessidade de muitos cirurgiões-dentistas ainda não conhecerem este medicamento, bem como a sua utilização em pacientes sistemicamente comprometidos, sendo que ele tem benefícios de grande relevância para bons resultados clínicos. Dessa forma, o objetivo deste trabalho é buscar evidências sobre suas aplicações na odontologia diante de tantas doenças hematológicas que merecem atenção redobrada, principalmente, durante os procedimentos clínicos-cirúrgicos.

\section{Metodologia}

\subsection{Delineamento do estudo}

Trata-se de uma revisão da literatura na modalidade integrativa, partindo de uma interrogação a fim de nortear a busca eletrônica nas bases de dados, indagando-se: "Qual a efetividade do ácido tranexâmico quando utilizado em procedimentos odontológicos de pacientes sistemicamente comprometidos?" (Pereira et al., 2018).

\subsection{Estratégia de busca}

O levantamento bibliográfico foi realizado através de consulta em bases de dados relevante para a produção do conhecimento em odontologia: Scientific Electronic Library Online (SciELO), Medical Literature Analysis and Retrieval System Online (MEDLINE), Literatura Latino-Americana e do Caribe em Ciências da Saúde (LILACS) e Biblioteca Brasileira de Odontologia (BBO), utilizando cruzamentos com os seguintes descritores do vocabulário Descritores em Ciência da Saúde (DeCS): "Ácido Tranexâmico/Tranexamic Acid", "Doenças Sistêmicas/Systemic diseases" e "Odontologia/Dentistry" utilizando and/a para confrontar os termos boleanos.

\subsection{Critérios de inclusão e exclusão}

Como critérios de inclusão foram utilizados: estudos completos que descreviam a efetividade do uso do ácido tranexâmico na odontologia, contemplando pelo menos um dos descritores no título ou resumo, publicados em português e inglês entre os anos de 2009 a 2019.

Foram excluídos da amostra os artigos que não estavam disponíveis na íntegra, assim como artigos repetidos, editoriais, relatos de casos, artigos que relatavam sobre o uso do ácido em outras áreas da saúde, artigo em outros idiomas e estudos que não apresentassem relação com o objeto estudado.

\subsection{Seleção dos artigos}

Após as buscas eletrônicas, foi realizada a leitura dos resumos de artigos, selecionando os trabalhos em consonância aos critérios de inclusão, posteriormente, descartando todos que estava dentro do parâmetro de exclusão. Em seguida, os mesmos foram obtidos integralmente.

Alguns dos estudos selecionados foram analisados e organizados através da construção de uma tabela, do qual se obteve as seguintes informações: autor/ano, tipo de estudo, objetivo, resultados e conclusão do artigo.

\section{Resultados e Discussão}

Dessa forma, levando em consideração os modelos propostos na metodologia deste estudo, foram obtidos 118 artigos na busca inicial. Após a seleção manual, por meio de leitura dos títulos e resumos, foram descartados aqueles que não se encaixavam nas propostas deste estudo conforme fluxograma abaixo onde se detalha o processo de busca dos artigos científicos (Figura 1). 
Research, Society and Development, v. 10, n. 7, e3110716119, 2021

(CC BY 4.0) | ISSN 2525-3409 | DOI: http://dx.doi.org/10.33448/rsd-v10i7.16119

Figura 1 - Processo de busca dos artigos científicos.

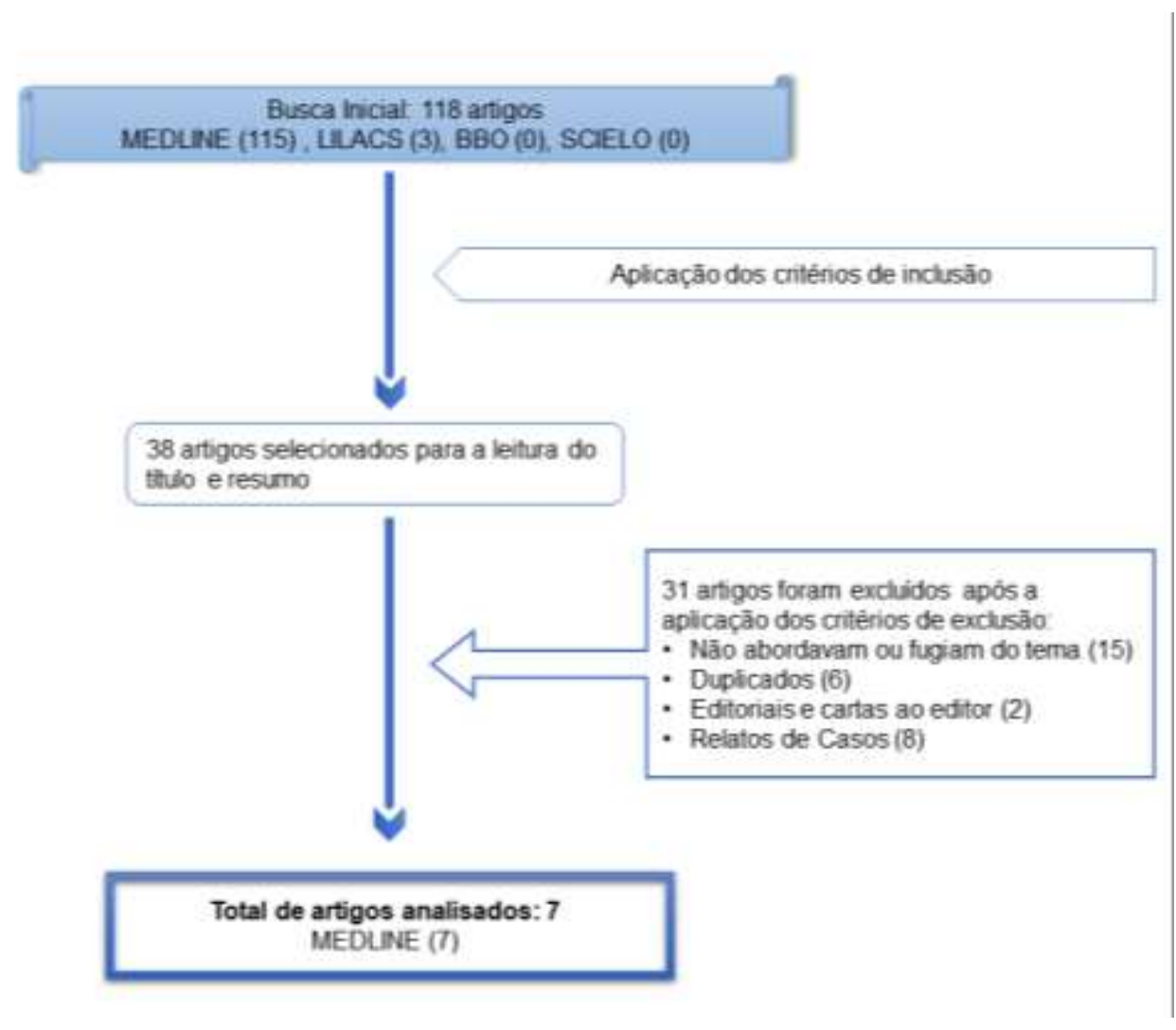

Fonte: Autores.

Por conseguinte, com base neste processo, foram selecionados 7 artigos científicos, abordando a eficácia do ácido tranexâmico em procedimentos odontológicos em pacientes com comprometimento sistêmico, sendo artigos nacionais ou internacionais, o último apenas se estivessem em inglês. No Quadro 1, temos a consolidação das principais informações contidas em cada artigo e está ordenado de acordo com o ano de publicação. 
Quadro 1 - Descrição dos dados dos estudos incluídos na revisão integrativa.

\begin{tabular}{|c|c|c|c|c|}
\hline AUTOR/ANO & $\begin{array}{l}\text { TIPO DE } \\
\text { ESTUDO }\end{array}$ & OBJETIVO & RESULTADOS & CONCLUSÃO \\
\hline Madrid \& Sanz, 2009 & $\begin{array}{l}\text { Revisão } \\
\text { sistemática }\end{array}$ & $\begin{array}{l}\text { Avaliar os riscos } \\
\text { (tromboembólicos e } \\
\text { hemorrágicos) de um } \\
\text { paciente com terapia de } \\
\text { anticoagulação oral } \\
\text { (OAT) submetido a } \\
\text { terapia de implante e } \\
\text { fornecer um protocolo } \\
\text { de manejo para os } \\
\text { mesmos. }\end{array}$ & $\begin{array}{l}\text { Os resultados destes estudos são } \\
\text { muito homogêneos, relatando } \\
\text { sangramento menor em poucos } \\
\text { pacientes, sem uma diferença } \\
\text { significativa entre os pacientes com } \\
\text { OAT que continuam com os } \\
\text { antagonistas da vitamina K versus os } \\
\text { pacientes que pararam este } \\
\text { medicamento antes da cirurgia. } \\
\text { Estes eventos pós-operatório do } \\
\text { sangramento foram controlados } \\
\text { somente com as medidas } \\
\text { hemostáticas locais: os bochechos } \\
\text { do ácido tranexâmico, as esponjas da } \\
\text { gelatina e a aplicação das gazes da } \\
\text { celulose eram eficazes. }\end{array}$ & $\begin{array}{l}\text { A descontinuação da AOT } \\
\text { não é recomendada para a } \\
\text { cirurgia oral menor, tal como } \\
\text { a única extração do dente ou a } \\
\text { colocação do implante, } \\
\text { contanto que esta não envolva } \\
\text { corrupções do osso autógeno, } \\
\text { aletas extensivas ou } \\
\text { preparações do osteotomia } \\
\text { que estendem fora do } \\
\text { envelope ósseo. As evidências } \\
\text { não sustentam que a } \\
\text { colocação de implantes } \\
\text { dentários em pacientes com } \\
\text { OAT é contraindicada. }\end{array}$ \\
\hline Perdigão et al., 2012 & $\begin{array}{l}\text { Estudo } \\
\text { observacional } \\
\text { prospectivo }\end{array}$ & $\begin{array}{l}\text { Avaliar a incidência de } \\
\text { sangramento pós- } \\
\text { operatório após } \\
\text { extração dentária em } \\
\text { candidatos ao } \\
\text { transplante hepático e a } \\
\text { eficácia da Associação } \\
\text { de ácido tranexâmico e } \\
\text { esponjas hemostáticas } \\
\text { absorvíveis. }\end{array}$ & $\begin{array}{l}\text { Nos } 23 \text { pacientes incluídos neste } \\
\text { estudo, } 84 \text { extrações simples foram } \\
\text { realizadas durante } 35 \text { procedimentos } \\
\text { cirúrgicos odontológicos. O } \\
\text { sangramento pós-operatório ocorreu } \\
\text { durante } 1 \text { procedimento ( } 2,9 \% \text { ), e a } \\
\text { pressão local com gaze era eficaz } \\
\text { para conseguir a hemostasia. Não foi } \\
\text { encontrada significância estatística } \\
\text { no tempo de hemostasia entre os } 2 \\
\text { grupos. }\end{array}$ & $\begin{array}{l}\text { Este estudo encontrou um } \\
\text { baixo risco de sangramento } \\
\text { para extrações do dente nos } \\
\text { pacientes com cirrose de } \\
\text { fígado. Não foram necessárias } \\
\text { transfusões sanguíneas e, no } \\
\text { caso do sangramento pós- } \\
\text { operatório, o uso de medidas } \\
\text { hemostáticas locais foram } \\
\text { satisfatórias. }\end{array}$ \\
\hline Broekema et al., 2014 & $\begin{array}{l}\text { Estudo } \\
\text { observacional } \\
\text { prospectivo }\end{array}$ & $\begin{array}{l}\text { Avaliar a incidência do } \\
\text { sangramento após } \\
\text { operações dentoalveolar } \\
\text { em pacientes, que não } \\
\text { estavam tomando } \\
\text { anticoagulantes }\end{array}$ & $\begin{array}{l}\text { Nenhum paciente desenvolveu um } \\
\text { sangramento severo que exigia a } \\
\text { intervenção. Sete pacientes (7\%) } \\
\text { tomar anticoagulantes desenvolveu } \\
\text { sangramentos pós-operatório } \\
\text { suaves. Os pacientes que tomam } \\
\text { antagonistas da vitamina K } \\
\text { relataram } 3 \text { episódios (9\%) em } \\
\text { comparação com } 4 \text { (6\%) no grupo } \\
\text { que toma inibidores da agregação de } \\
\text { trombócitos. Entre os doentes que } \\
\text { não tomam anticoagulantes, dois } \\
\text { (2\%) desenvolveram sangramento } \\
\text { leve. }\end{array}$ & $\begin{array}{l}\text { Todo o sangramento foi } \\
\text { controlado pelos próprios } \\
\text { pacientes com compressão } \\
\text { com gaze. Conclui-se que a } \\
\text { cirurgia dentoalveolar é } \\
\text { segura em pacientes tratados } \\
\text { com anticoagulantes, desde } \\
\text { que as condições descritas nas } \\
\text { diretrizes do ACTA sejam } \\
\text { atendidas. }\end{array}$ \\
\hline Van Galen et al., 2015 & $\begin{array}{l}\text { Revisão } \\
\text { Sistemática }\end{array}$ & $\begin{array}{l}\text { Avaliar a eficácia do } \\
\text { uso local ou sistêmico }\end{array}$ & $\begin{array}{l}\text { Em geral, os dois ensaios incluíram } \\
\text { um efeito benéfico do ácido }\end{array}$ & $\begin{array}{l}\text { Os estudos não nos permitem } \\
\text { concluir a eficácia definitiva }\end{array}$ \\
\hline
\end{tabular}




\begin{tabular}{|c|c|c|c|c|}
\hline & & $\begin{array}{l}\text { de agentes } \\
\text { antifibrinolíticos para } \\
\text { prevenir complicações } \\
\text { hemorrágicas em } \\
\text { pessoas com hemofilia } \\
\text { ou doença de von } \\
\text { Willebrand submetidas } \\
\text { a procedimentos orais } \\
\text { ou odontológicos }\end{array}$ & $\begin{array}{l}\text { tranexâmico e da EACA, } \\
\text { administrados sistemicamente, na } \\
\text { redução do número de sangramento, } \\
\text { na quantidade de perda de sangue e } \\
\text { na necessidade de concentrados de } \\
\text { fator de coagulação terapêutico. }\end{array}$ & $\begin{array}{l}\text { da terapêutica antifibrinolítica } \\
\text { em procedimentos orais ou } \\
\text { dentários em pessoas com } \\
\text { hemofilia. Não foram } \\
\text { identificados ensaios em } \\
\text { pessoas com doença de von } \\
\text { Willebrand. }\end{array}$ \\
\hline Vasconcellos et al., 2016 & $\begin{array}{l}\text { Revisão } \\
\text { Sistemática e } \\
\text { meta-analise }\end{array}$ & $\begin{array}{l}\text { Investigar a eficácia e } \\
\text { segurança do ácido } \\
\text { tranexâmico tópico } \\
\text { (ATX) para prevenir } \\
\text { sangramento pós- } \\
\text { operatório em pacientes } \\
\text { anticoagulados } \\
\text { submetidos a cirurgia } \\
\text { oral menor. }\end{array}$ & $\begin{array}{l}\text { Há um efeito protetor da ATX tópico } \\
\text { no sangramento após cirurgias orais } \\
\text { menores. A análise do subgrupo } \\
\text { revelou que ATX tópico era eficaz } \\
\text { em impedir o sangramento Pós- } \\
\text { cirúrgico comparado ao placebo e ao } \\
\text { ácido Épsilon-Aminocaproico. }\end{array}$ & $\begin{array}{l}\text { Evidências atualmente } \\
\text { disponíveis sugerem que a } \\
\text { irrigação do local cirúrgico } \\
\text { com ATX seguida de } \\
\text { enxaguatório bucal durante a } \\
\text { primeira semana de pós- } \\
\text { operatório é segura e pode } \\
\text { reduzir o risco de } \\
\text { sangramento após cirurgias } \\
\text { orais menores em pacientes } \\
\text { anticoagulados. }\end{array}$ \\
\hline Van Galen et al., 2019 & $\begin{array}{l}\text { Revisão } \\
\text { Sistemática }\end{array}$ & $\begin{array}{l}\text { Avaliar a eficácia dos } \\
\text { agentes } \\
\text { antifibrinolíticos para } \\
\text { prevenir complicações } \\
\text { hemorrágicas em } \\
\text { doentes com hemofilia } \\
\text { VWD submetidos a } \\
\text { procedimentos orais ou } \\
\text { dentários. }\end{array}$ & $\begin{array}{l}\text { Em geral, os dois ensaios incluíram } \\
\text { um efeito benéfico do ácido } \\
\text { tranexâmico e da EACA, } \\
\text { administrados sistemicamente, na } \\
\text { redução do número de sangramento, } \\
\text { na quantidade de perda de sangue e } \\
\text { na necessidade de concentrados de } \\
\text { fator de coagulação terapêutico. }\end{array}$ & 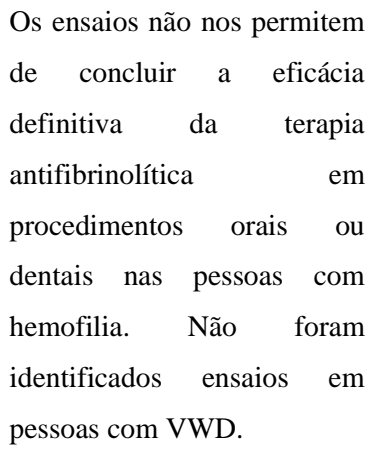 \\
\hline Ping et al., 2019 & $\begin{array}{l}\text { Revisão } \\
\text { Sistemática e } \\
\text { meta-analise }\end{array}$ & $\begin{array}{l}\text { Avaliar o papel da ácido } \\
\text { tranexâmico em } \\
\text { pacientes submetidos a } \\
\text { cirurgias nasais. }\end{array}$ & $\begin{array}{l}\text { Houve diferenças significativas na } \\
\text { perda de sangue (P <. 001), } \\
\text { qualidade do campo cirúrgico }(\mathrm{P}<\text {. } \\
\text { 001), avaliação do edema da parte } \\
\text { superior }(\mathrm{P}<.001) \text { e menor }(\mathrm{P}<\text {. } \\
001) \text { pálpebra, equimose superior (p } \\
\text { <. 001) e pálpebra inferior }(\mathrm{P}<.001) \\
\text { ao comparar o grupo ATX com o } \\
\text { grupo placebo. No entanto, a } \\
\text { diferença no tempo de operação }(\mathrm{P} \\
=. \text { 57) não foi significante entre os } \\
\text { dois grupos. }\end{array}$ & $\begin{array}{l}\text { O ATX perioperatório pode } \\
\text { reduzir a perda de sangue e } \\
\text { melhorar a qualidade do } \\
\text { campo cirúrgico durante a } \\
\text { cirurgia nasal, e foi auxiliada } \\
\text { por reduzir o edema e a } \\
\text { equimose após cirurgias } \\
\text { nasais, mas tem pouca } \\
\text { influência na redução do } \\
\text { tempo de operação. }\end{array}$ \\
\hline
\end{tabular}

Fonte: Autores.

Estudos recentes mostram que o número de pacientes com discrasias sanguíneas são cada vez mais recorrentes nos consultórios odontológicos, diante disso a utilização de medicamentos para hemostasias ou controle de hemorragias são frequentes e precisam ser estudados para sua correta indicação, uma vez que ainda há controvérsias quando se trata de pacientes 
que estão em terapia com anticoagulante ou pacientes que estão em terapia antifibrinolítica (Madrid \& Sanz, 2009; Perdigão et al., 2012).

Há estudos bem homogêneos em que demonstram resultados pouco expressivo quanto ao controle do sangramento de pacientes com terapia de anticoagulação oral (AOT), mesmo sendo eficaz medidas hemostáticas locais para conter o sangramento como a utilização de bochechos do ácido tranexâmico e esponjas de gelatina, sem uma diferença significativa entre quem continuaram com os antagonistas da vitamina $\mathrm{K}$ versus os pacientes que pararam este medicamento antes da cirurgia (Madrid \& Sanz, 2009; Broekema et al., 2014).

Todavia, sabe-se que interromper a AOT não é recomendada para cirurgias orais mesmo que seja uma simples exodontia, contanto que não tenha um grande envolvimento que se estenda fora do envelope ósseo como as osteotomias, pois não há evidencias que comprovem a contraindicação de extrações, assim como também colocação de implantes em pacientes em terapia anticoagulante (Madrid \& Sanz, 2009).

Dentre as doenças que causam distúrbios no processo de coagulação sanguínea, estão a hemofilia e a doença de Von Willebrand, ambas são doenças hemorrágicas genéticas e hereditárias, porém a primeira é mais frequente em homens devido a deficiência da atividade de fatores de coagulação sanguínea VIII (hemofilia A) ou IX (hemofilia B) e a segunda causada por uma diminuição ou uma disfunção da proteína chamada de fator de von Willebrand (FvW) (Ferrari et al., 2015; Farrkh et al., 2016; Silva et al., 2012; Van Galen et al., 2015).

Esses dois tipos de doenças podem ser utilizados agentes antifibrinolíticos como o ácido tranexâmico para prevenir complicações hemorrágicas em procedimentos odontológicos. Em geral, este medicamento tem efeito benéfico quando utilizado de forma sistêmica, pois reduz do número de sangramento, de perda de sangramento no transoperatório, bem como a necessidade de concentrados de fator de coagulação terapêutico pós-cirúrgicos (Van Galen et al., 2015).

Entretanto, os estudos não nos permitem ainda concluir a eficácia definitiva da terapêutica antifibrinolítica em procedimentos orais ou dentários em pessoas com hemofilia como também em portadores de doença de Von Willebrand, pois o número de ensaios controlados aleatorizados e amostras ainda são pequenos, portanto pouco expressivos (Van Galen et al., 2015; Ripollés-de Ramón et al., 2014).

No que se refere a efetividade e segurança do ácido tranexâmico para prevenção de sangramento pós-operatório em pacientes que fazem uso de anticoagulantes submetidos a cirurgia oral menor, pode-se afirmar que evidências atualmente disponíveis sugerem que a irrigação do local cirúrgico com ATX seguida de enxaguatório bucal durante a primeira semana de pós-operatório é efetiva e segura, pois reduz consideravelmente o risco de sangramento após cirurgias orais menores, garantindo assim um prognóstico favorável (Ripollés-de Ramón et al., 2014; Milani et al., 2017; Karimi et al., 2012).

O ácido tranexâmico pode ser também utilizado de diversas maneiras e formas farmacêuticas em ambiente hospitalar, bem como a beira leito, seja ele em gel, comprimido, colutório ou injetável, tendo efeito local ou sistêmico. Dessa forma, o mesmo pode ser utilizado em cirurgias bucomaxilofaciais, com envolvimento de ossos da face, a exemplo, os nasais (Ripollésde Ramón et al., 2014; Donat et al., 2013; Ping et al., 2019). Nesses tipos de cirurgias o ATX perioperatório pode reduzir a perda de sangue e melhorar a qualidade do campo cirúrgico durante a cirurgia, além de auxiliar na redução do edema e da equimose após cirurgias nasais, mas tem pouca influência na redução do tempo do procedimento cirúrgico (Ping et al., 2019; Karimi et al., 2012; Vale et al., 2017).

Sabe-se que os pacientes tratados com anticoagulantes orais têm maior susceptibilidade a sangramento, e, portanto, qualquer procedimento médico cirúrgico e, especialmente, a cirurgia oral, requer uma abordagem terapêutica que minimize os efeitos do sangramento nesses pacientes. Nesse sentido, pesquisas mostram que os resultados indicam que a aplicação do gel de ácido tranexâmico é muito eficaz para consistência e manutenção no local de ação e mostra sua eficácia como um material prócoagulante. Os principais benefícios em relação a aplicação de uma solução gel de ácido tranexâmico em pacientes com 
anticoagulantes orais é a melhora no tempo de cicatrização e o tempo de sangramento nas primeiras 48-72 horas (Ripollés-de Ramón et al., 2014; Capalbo et al., 2012).

$\mathrm{Na}$ literatura há estudos também que apontam a utilização do papel do ácido tranexâmico na geração do coágulo sanguíneo, formação óssea e osseointegração de implantes dentários. Estes estudos concluíram que os resultados dentro da implantodontia são positivos, uma vez que sugerem o favorecimento da geração de coágulo e osteointegração pela ação do ácido tranexâmico, o que acaba aumentando a capacidade osteogênica dos implantes de titânio (Capalbo et al., 2012).

Nesse contexto, sabe-se que os agentes antifibrinolíticos, tais como o ácido tranexâmico, inibem a fibrinólise e, consequentemente, impedem ou diminuem a formação dos produtos de degradação da fibrina e do fibrinogênio. Portanto, este ácido a 5\% pode ser prescrito para lavagem da cavidade bucal após exodontias ou na forma de pasta. Outra característica interessante é que o ácido tranexâmico é solúvel em compostos a base de água, podendo ser feito o preparo da solução com um comprimido de 500mg triturado e misturado a soro fisiológico, logo em seguida devem ser filtrados os excipientes insolúveis para resultar em solução límpida (Rezende et al., 2014; Vale et al., 2017).

Em situações que se faz o preparo com soro fisiológico deve-se colocar em gaze ou mesmo diretamente sobre a ferida cirúrgica para que assim aconteça o equilíbrio do mecanismo de hemostasia, como um papel fundamental no curso natural da reparação dos tecidos, possibilitando assim a formação do coagulo e, consequentemente, a completa cicatrização (Rezende et al., 2014; Monteiro et al., 2016).

Desse modo, faz-se necessário que o Cirurgião-Dentista tenha conhecimento dos diferentes meios de utilização e prescrição do uso do ácido tranexâmico para que assim obtenha bons resultados, tendo em vista o leque de possibilidades de aplicação desse ácido em diversas situações clínicas de pacientes sistemicamente comprometidos.

\section{Considerações Finais}

Nos estudos analisados, percebe-se que o ácido tranexâmico quando bem indicado promove ótimos benefícios frente aos problemas de pacientes com coagulopatias, sendo o maior deles o controle no sangramento e a melhora no processo de cicatrização, uma vez que estes pacientes estão sujeitos a complicações hemorrágicas que podem até mesmo levar ao óbito. Além disso, vale ressaltar que pacientes com transtornos de coagulação e que também fazem uso de anticoagulantes ou antiagregantes plaquetários requerem maior atenção e cuidados especiais durante a prática odontológica.

Logo, fazem-se necessários os conhecimentos relacionados aos meios de hemostasia eficazes durante os procedimentos clínico-cirúrgicos, inclusive os que envolvem o uso do ácido tranexâmico. Pois, este medicamento tem se mostrado efetivo ao reduzir o risco de hemorragia durante cirurgias orais e tratar hemorragias associadas à fibrinólise excessiva, mostrando-se seguro e profícuo nos procedimentos odontológicos de pacientes sistemicamente comprometidos, tais como: as cirurgias orais menores, reparos ósseos periimplantares, implantes dentários, manejo de lesões císticas e raspagem subgengivais, dentre outros. Em virtude da importância do Ácido tranexâmico no controle dos processos hemorrágicos, vislumbramos como perspectiva de trabalhos futuros um estudo de caso controle na clínica cirúrgica odontológica.

\section{Referências}

Broekema, F. I., Van Minnen, B., Jansma, J \& Bos, R. R. (2014). Risk of bleeding after dentoalveolar surgery in patients taking anticoagulants. Br. j. oral maxillofac. surg. 52(3):e15-9.

Capalbo, B. C., Alves-Rezende, M. C., Louzada, M. J. \& Alves-Claro, A. P. (2012). Geração do coágulo sanguíneo, formação óssea e osseointegração de implantes dentários: ação do ácido tranexâmico. Arch. health invest. 1(Suppl. 1).

Cury, M. T., Gonçalves, V. M., Oliveira, J. A., Lisboa Filho, P. N. \& Rezende, M. A. (2015). P-o60 Estudo da osseointegração em ratos diabéticos. Efeito da associação adesivo fibrínico/ácido tranexâmico. Arch. health invest. 4(1):311. 
Da Silva, K. T., Júnior, E. Á., Érnica, N. M., Griza, G. L. \& Pasqualotto, L. N. (2016). Abordagem cirúrgico-odontológica em paciente com trombastenia de glazmann: relato de caso. Rev. Uningá Review. 27(3):33-5.

De Vasconcellos, S. J., Santos, T. S., Reinheimer, D. M., Faria-e-Silva, A. L., de Melo, M. D. \& Martins-Filho, P. R. (2017). Topical application of tranexamic acid in anticoagulated patients undergoing minor oral surgery: A systematic review and meta-analysis of randomized clinical trials. J. oral cranio-maxillofac. surg. 45(1):20-6.

Donat, M. G., Alexander, L. V., Yang, H., Durre, I., Vose, R., Dunn, R. J, et al. (2013). Updated analyses of temperature and precipitation extreme indices since the beginning of the twentieth century: The HadEX2 dataset. J. of Geophysical Rese. Atmosphe. 118(5):2098-118.

Farrkh, A., Garrison, E. \& Closmann, J. J. (2016). Dental surgical management of the patient with hemophilia. Gen Dent. 64(4):14-7.

Ferrari, D. C., Machado, L., Menegais, K. \& Coelho, V. K. (2015). Fármacos que afetam a circulação sanguínea: fibrinolíticos e antifibrinolíticos. Ação Odonto. $3(1): 1-13$.

Karimi, A., Mohammadi, S. S. \& Hasheminasab, M. (2012). Efficacy of tranexamic acid on blood loss during bimaxilary osteotomy: A randomized double blind clinical trial. Saudi j. of anaesthesia. 6(1):41-5.

Kuhn-Dall'Magro, A., Ribeiro, A. A., Shenkel, A., Samuelsson, M., Studzinski, M. S. \& Almeida, D. (2011). Manejo odontológico de pacientes com coagulopatias-revisão de literatura e relato de caso: síndrome de Bernard Soulier. Rev. Fac. Odontol. Univ. UPF. 16(2):1-7.

Madrid, C. \& Sanz, M. (2009). What influence do anticoagulants have on oral implant therapy? A systematic review. Clin. oral implants res. 96-106.

Milani, C. L., Kolodziejwski, W. T., de Godoy, I. P., Salha, R. A., Remor, F. \& Nardi, A. (2017). Cuidados no tratamento odontológico em pacientes anticoagulados. Ação Odonto. https://portalperiodicos.unoesc.edu.br/acaodonto/article/view/13552.

Monteiro, et al. (2016). Osteíte alveolar: fatores de risco e modalidades de tratamento. Braz. J. Sur. Clin. Res. - BJSCR. 15(3), 68-72.

Nematullah, A., Alabousi, A., Blanas, N., Douketis, J. D. \& Sutherland, S. E. (2009). Dental surgery for patients on anticoagulant therapy with warfarin: a systematic review and meta-analysis. J. Can. Dent. Assoc. 75(1).

O'Leary, J. G., Yachimski, P. S. \& Friedman, L. S. (2009). Surgery in the patient with liver disease. Clin. liver dis. 13(2):211-31.

Perdigão, J. P., de Almeida, P. C., Rocha, T. D., Mota, M. R., Soares, E. C., Alves, A. P, et al. (2012). Postoperative bleeding after dental extraction in liver pretransplant patients. Int. j. oral maxillofac. surg. 70(3):e177-84.

Pereira A. S. et al. (2018). Metodologia da pesquisa científica. UFSM. 5.3)

Koche, J. C. (2011). Fundamentos de metodologia científica. Petrópolis: Vozes. http://www.brunovivas.com/wp-content/uploads/sites/10/2018 /07/K\%C3\%B6che-Jos\%C3\%A9-Carlos0D0AFundamentos-de-metodologia-cient\%C3\%ADfica-_teoria-da0D0Aci\%C3\%AAncia-e-inicia\%C3\%A7\%C3\%A 3o-\%C3\%A0-pesquisa.pdfhttps://repositorio.ufsm.br/bitstream/handle/1/15824/Lic_Computacao_Metodologia-Pesquisa-Cientifica.pdf?sequence=1 5.4)

Ping, W. D., Zhao, Q. M., Sun, H. F., Lu, H. S. \& Li, F. (2019). Role of tranexamic acid in nasal surgery: A systemic review and meta-analysis of randomized control trial. Medicine. 98(16): e15202.

Ragab, S. M. \& Hassanin, M. Z. (2010). Optimizing the surgical field in pediatric functional endoscopic sinus surgery: a new evidence-based approach. Otolaryngol. head neck surg. 142(1):48-54.

Rezende, $\mathrm{M}$ et al. (2014). Efeito do ácido tranexâmico associado à cola de fibrina sobre o reparo ósseo: estudo histológico em ratos. Arch Health Invest. 3(4): $59-65$.

Ripollés-de Ramón, J., Muñoz-Corcuera, M., Bravo-Llatas, C. \& Bascones-Martínez, A. (2014). Aplicación de un gel de ácido tranexámico en pacientes tratados con anticoagulantes orales. Med. Clínic. 143(11):484-8.

Ripollés-de Ramón, J., Muñoz-Corcuera, M., Bravo-Llatas, C. \& Bascones-Martínez, A. (2014). Aplicación de un gel de ácido tranexámico en pacientes tratados con anticoagulantes orales. Medic Clínic. 143(11):484-8.

Silva, S. C., Silva, E. S., Menezes, K. T., Lira Júnior, R. \& Brito, R. L. (2012). Tratamento periodontal de paciente hemofílico. Rev. bras. ciênc. saúde;16(2):2438.

Vale, G. (2017). Anticoagulação oral em pacientes submetidos a tratamento médico-dentário: proposta de normas terapêuticas. Instituto Superior De Ciências Da Saúde Egas Moniz- Mestrado Integrado Em Medicina Dentária, Repositório Comum; Portugal.

Van Galen, K. P., Engelen, E. T., Mauser-Bunschoten, E. P., Van Es, R. J. \& Schutgens, R. E. (2015). Antifibrinolytic therapy for preventing oral bleeding in patients with haemophilia or Von Willebrand disease undergoing minor oral surgery or dental extractions. Cochrane Database Syst Rev. (12): CD011385. 10.1002 / 14651858.CD011385.pub2.

Van Galen, K. P., Engelen, E. T., Mauser-Bunschoten, E. P., Van Es, R. J. \& Schutgens, R. E. (2019). Antifibrinolytic therapy for preventing oral bleeding in patients with haemophilia or Von Willebrand disease undergoing minor oral surgery or dental extractions. Cochrane Database of Systematic Reviews Issue 4. 10.1002/14651858.CD011385.pub3. 